\title{
Maximizing the Adequacy of Medication Treatment in Controlled Trials and Clinical Practice: STAR*D Measurement-Based Care
}

\author{
Madhukar H Trivedi*, , A John Rush', Bradley N Gaynes ${ }^{2}$, Jonathan W Stewart ${ }^{3}$, Stephen R Wisniewski ${ }^{4}$, \\ Diane Warden', Louise Ritz ${ }^{5}$, James F Luther ${ }^{4}$, Diane Stegman', Joanne Deveaugh-Geiss ${ }^{2}$ and \\ Robert Howland ${ }^{6}$
}

'Department of Psychiatry, University of Texas Southwestern Medical Center at Dallas, Dallas, TX, USA; ${ }^{2}$ Department of Psychiatry, University of North Carolina School of Medicine, Chapel Hill, NC, USA; ${ }^{3}$ Department of Psychiatry, College of Physicians and Surgeons, Columbia University, New York, NY, USA; ${ }^{4}$ Epidemiology Data Center, Graduate School of Public Health, University of Pittsburgh School of Medicine, Pittsburgh, PA, USA; ${ }^{5}$ National Institute of Mental Health, Bethesda, MD, USA; ${ }^{6}$ Department of Psychiatry, University of Pittsburgh School of Medicine,

Pittsburgh, PA, USA

\begin{abstract}
The success of well-developed protocols has been limited in real-world practice, where even effective strategies have not been sufficient to meet patient needs in routine clinical care owing to Axis I and III comorbidities. The Sequenced Treatment Alternatives to Relieve Depression (STAR*D) trial required that antidepressant medication treatment be optimal regarding dose and duration, yet accommodate flexibility to ensure safety given the wide range of comorbid general medical and psychiatric disorders allowed in the trial. The objective of this study was to develop a measurement-based care (MBC) approach and an automated feedback system to ensure adequate and safe antidepressant treatment delivery suitable for both clinical research and routine practice. Ratings of depressive symptom severity and side-effect frequency, intensity, and burden were obtained at each treatment visit using the MBC system that (I) guided medication dose adjustments and treatment duration, (2) documented clinician adherence to treatment recommendations, and (3) provided prompt feedback to clinicians to enhance appropriate treatment decisions. Physician adherence to protocol-specific treatment was monitored based on measured symptoms and side-effect burden, and dose and duration of antidepressant at each critical decision point during the acute phase treatment of major depression. Feedback was provided at the point of care by the clinical coordinators, assisted by Web-based reports following each treatment visit. On the basis of the first treatment step with citalopram, over $85 \%$ of treatment encounters had appropriate fidelity to recommendations. Most deviations from treatment recommendations occurred late in treatment and were often justifiable. MBC proved to be feasible and effective in busy primary and psychiatric settings. This approach signals a paradigm shift toward the use of measurement-based clinical decisions, both at the point of care and following each visit, to deliver optimal pharmacotherapy for depression.
\end{abstract}

Neuropsychopharmacology (2007) 32, 2479-2489; doi:I 0.1038/sj.npp. I301390; published online 4 April 2007

Keywords: depression algorithm; physician decision support; symptom monitoring; adequate antidepressant treatment; maximizing pharmacotherapy

\section{INTRODUCTION}

The treatment of depression in clinical practice is often not in accord with evidence-based recommendations or guidelines. Patients are undertreated with respect to dose and duration of antidepressant medication (Katon et al, 1995;

*Correspondence: Dr MH Trivedi, Department of Psychiatry, University of Texas Southwestern Medical Center at Dallas, 5323 Harry Hines Blvd., Dallas, TX 75390-9119, USA, Tel: + I 214648 0188, Fax: + 12146480167 ,

E-mail: madhukar.trivedi@utsouthwestern.edu

Received 17 November 2005; revised 6 February 2007; accepted 8 February 2007
Kessler et al, 2003; Simon et al, 1993; Trivedi et al, 2004a; Trivedi and Kleiber, 2001) or have inadequate follow-up (Ford, 2000), particularly during the critical initial stages of treatment (Ford, 2000; Lin et al, 1995; Trivedi and Baker, 2001). Consequently, desired outcomes (full symptomatic remission and return to premorbid levels of functioning) are often not achieved.

Even in guideline-driven practice, the clinical treatment of depression is often associated with wide variations among practitioners (eg in doses used and in the duration of the treatment trials). Clinicians often change from one antidepressant to another too quickly or conversely, conduct an unnecessarily prolonged treatment trial with 
an obviously unsuccessful medication or psychotherapy (Rush et al, 2003a; Trivedi et al, 2006b). In addition, practitioners differ in how they assess the outcomes of treatment (symptoms, function, side-effect frequency and burden). Global judgments are often used instead of specific symptom assessments, even though the former are less accurate (Biggs et al, 2000). These procedural differences lead to wide variability in treatment implementation and likely also result in wide variations in outcomes in typical practice.

Recent studies have shown that improving the care delivery system improves outcomes for depressed patients in both primary (Katon et al, 1999, 2004; Unützer et al, 2002) and specialty care (Trivedi et al, 2004b) settings. However, even with enhanced treatment involving depression care specialists or clinical coordinators, remission rates often remain low (15-30\%; Rush et al, 2004b). Wellestablished findings indicate that specific recommendations to guide treatment decisions are most effective when they are individualized and are provided while the clinical decision is being made (Trivedi et al, 2000, 2002, 2004a). Treatment trials for the management of major chronic medical illnesses, such as diabetes mellitus, hypertension, and asthma utilize laboratory as well as symptom and function measures in research settings that are readily usable in clinical practice. To our knowledge, however, no system to provide specific feedback or prompts related to symptoms, side effects, and recommended tactics (ie when and by how much to change the dose) during treatment has been successfully used in a large clinical trial for patients with psychiatric disorders. We are also not aware of any attempts to develop and implement easy-to-use, measurement-based research tools for feedback that may be readily transferred to clinical practice when treating these disorders.

The implementation of antidepressant medication in the Sequenced Treatment Alternatives to Relieve Depression $\left(\mathrm{STAR}^{\star} \mathrm{D}\right)$ trial (Fava et al, 2003; Rush et al, 2004a) required that antidepressant medication treatments be fully adequate in dose and duration, yet safe and well tolerated. It was essential that adequate treatment is delivered at each treatment step before declaring treatment failure and proceeding to the next step.

The STAR ${ }^{\star} \mathrm{D}$ trial enrolled 4041 broadly representative outpatients with nonpsychotic major depressive disorder (MDD) from 'real-world' clinical practices. A substantial proportion of participants, therefore, had concomitant general medical conditions $(52.8 \%)$ (Yates et al, 2004), active substance abuse/dependence (20\%) (Davis et al, 2005), or other Axis I disorders (63\%) (Rush et al, 2005). The trial was conducted in primary and psychiatric care settings by clinicians as part of their daily treatment of depressed outpatients rather than in research settings. In this context, it was not only essential to optimally dose medications concordant with the $\mathrm{STAR}^{\star} \mathrm{D}$ treatment protocol, but also to individualize starting doses and dose escalations to minimize side effects, maximize safety, and optimize the chances of therapeutic benefit for each participant.

Given wide practice variations and the variable implementation of guideline-driven care, $\mathrm{STAR}^{\star} \mathrm{D}$ chose not to accept routine practice. Instead, we provided measurement- based care (MBC). Three specific objectives of $\mathrm{MBC}$ for $\operatorname{STAR}^{\star} \mathrm{D}$ were identified: (1) check adherence on a systematic and ongoing basis to identify deviations from the protocol and provide an opportunity for rapid corrective action, (2) provide documentation of clinician adherence to the manualized treatments that could be cited in study publications, and (3) demonstrate the feasibility of using a clinician feedback and prompt system that includes automated methods of improving adherence to a manualbased, clinically relevant treatment algorithm, and that can be implemented in routine clinical practices.

Specific recommendations for dosing tactics were provided in the form of a treatment manual. Unlike standard double-blind efficacy trials for depression that provide rigid predefined doses or unspecified 'flexible' dosing, the $\mathrm{STAR}^{\star} \mathrm{D}$ treatment protocol was designed for adaptation to specific clinical situations to define optimal care for individual participants at specified times. A Clinical Research Coordinator (CRC) monitored and prompted the treatment decisions with postvisit monitoring of care conducted through conference calls and a Web-based system. This method was designed to ensure that true treatment resistance was present before proceeding to the next treatment step. This design (sometimes called a 'hybrid' design; Rush et al, 1998; Rush, 1999) engages representative patients and providers, but enhances routine care. Therefore, the results are generalizable to reasonably well-delivered care of such patients. Hence, the STAR ${ }^{\star} \mathrm{D}$ monitoring scheme provided feedback to the clinical team (both the physician and the CRC) to ensure that the pharmacotherapies were delivered at adequate doses and for adequate durations, as gauged by the symptomatic status and side-effect severity and burden at the visits critical to making appropriate decisions (eg weeks 2, 4, 6, 9, and 12 visits). Clinically useful tools were developed, that promoted the use of maximally effective, well-tolerated medication dosages for a sufficient time period to ensure that the recognition of treatment failure was neither premature nor inappropriately delayed. These easy-to-use tools and methods were designed to provide feedback to and corrective action by physicians, and are easily transferable to routine practice.

We have recently described the clinical outcomes following the application of MBC in the first two levels of $\mathrm{STAR}^{\star} \mathrm{D}$. Use of citalopram in level-1 treatment led to mean exit doses of slightly more than $40 \mathrm{mg} /$ day and an average of five visits over 10 weeks (Trivedi et al, 2006b). Application of the MBC approach led to similarly aggressive dosing and duration for those not remitting and moving on to level-2 treatment, whether the intervention was pharmacologic augmentation of citalopram (Trivedi et al, 2006a) or switching to a different antidepressant (Rush et al, 2006). The specifics of the MBC approach, however, have yet to be described fully.

This paper describes the measurement-based, decisionmaking system and procedures, including (1) the dissemination and implementation of decision support tools at the point of care and (2) a technology-based physician feedback system (PFS) used following each treatment visit. These methods are applicable to both routine practice and to effectiveness trials when there is a need to enhance the consistency of care across providers. 


\section{MATERIALS AND METHODS}

\section{STAR $^{\star D}$ Study Methods}

Study description. The methods of STAR ${ }^{*} \mathrm{D}$ have been detailed elsewhere (Fava et al, 2003; Rush et al, 2004a). $\mathrm{STAR}^{*} \mathrm{D}$ was designed to define prospectively which of several treatments were most effective for outpatients with nonpsychotic MDD who have an unsatisfactory clinical outcome to an initial and, if necessary, subsequent treatment(s). The diagnosis of MDD was established clinically and confirmed with a checklist from the Diagnostic and Statistical Manual of Mental DisordersText Revision (DSM-IV TR) (American Psychiatric Association, 2000). Eligible and consenting participants were treated with a selective serotonin reuptake inhibitor (SSRI), after which those with an adequate clinical response could enter a 12-month naturalistic follow-up phase. Those without such a response could enter one or more subsequent randomized clinical trials.

Organization of $S T A R^{\star} D$. The $\operatorname{STAR}^{\star} \mathrm{D}$ infrastructure includes the National Coordinating Center in Dallas, the Data Coordinating Center in Pittsburgh, and 14 Regional Centers (RCs) across the United States. Each RC oversaw implementation of the protocol at two to four primary or specialty care clinical sites in either the public or private sector. Nearly half of the sites were primary care settings.

The CRCs at each clinical site were trained and certified in the implementation of the treatment protocol and in data collection methods. CRCs worked closely with the participants and clinicians, functioned as study coordinators, and provided a liaison between clinical sites and RCs. Research Outcomes Assessors, who were masked to treatment assignment, collected symptom ratings through telephone interviews. Additional research outcomes were collected by an automated interactive voice response telephone interview (Kobak et al, 1996, 1999; Mundt, 1997).

Clinical sites and participants. Clinical sites were identified based on the availability of a large number of depressed outpatients, the level of available administrative support, and the availability of clinicians and an ethnically representative patient population. To increase generalizability to patients seen in real-world settings, the study enrolled self-declared outpatients already seeking medical care in the clinical setting. Advertising for subjects was not permitted. Further, exclusion criteria were minimal: participants with bipolar disorder, psychotic disorder or symptoms (lifetime), or a primary diagnosis of obsessive compulsive or eating disorder were excluded. Patients with general medical conditions that contraindicated the use of medications used in the first two levels of the study or with substance dependence requiring inpatient detoxification were also excluded. Suicidal patients were eligible, except those requiring immediate hospitalization. Participants had to be 18-75 years of age and have a baseline 17-item Hamilton Rating Scale for Depression ( $\mathrm{HRSD}_{17}$ ) (Hamilton, $1960,1967)$ score $\geqslant 14$. Participants could not be breastfeeding or pregnant at study entry, or planning to be so in the subsequent 9 months. Participants were excluded if they had previously not responded in the current episode to an adequate trial of any treatment used in the first two levels of the study. All participants signed an informed consent before being assessed for study entry at the clinical site.

Study protocol. All STAR ${ }^{\star} \mathrm{D}$ participants received an initial trial of up to 14 weeks of the SSRI citalopram and all other medications used in the sequential treatment steps were provided to the patients through a pharmacy card (level 1), with flexible dose titration to $60 \mathrm{mg} /$ day. Participants who achieved remission or who had at least a response could proceed to a naturalistic, 12-month follow-up with periodic clinical visits, as well as monthly study evaluations.

Participants without sufficient symptomatic benefit, either owing to intolerance or lack of efficacy on the citalopram, were eligible for randomization across four switch options (citalopram to sertraline, bupropion-SR, venlafaxine-XR, or cognitive therapy) and three augment options (citalopram plus bupropion-SR, buspirone, or cognitive therapy). Participants were given some preference in opting out of certain strategies. They were allowed to eliminate certain treatment strata such that they could opt to reject augmentation and then would be randomized to switch or reverse. However, they were not allowed to opt for a particular medication (eg bupropion-SR). These options were selected both because of their close resemblance to current clinical practice and because they address specific common questions (eg when switching agents from an SSRI, is it better to select an antidepressant from another class or the same class?). Participants whose depression failed to remit at level 2 were offered participation in subsequent levels $2 \mathrm{~A}$ (for those treated with cognitive therapy) or 3 , and 4 as necessary. Each level lasted approximately 12 weeks. At the end of each level, those with a satisfactory response could proceed to the 12-month follow-up. Subsequent treatment levels included medications often used with resistant depression, but with potentially higher levels of side effects or greater degrees of difficulty in their use (eg lithium and thyroid augmentation, a tricyclic antidepressant, and a monoamine oxidase inhibitor).

\section{Pharmacotherapy Implementation and Monitoring Procedures}

To accomplish the objectives of ensuring maximally tolerated trials of study medications for an adequate period of time and allowing for flexibility (eg slower dose increase in the context of higher side effects, lower starting dose owing to age or comorbid general medical condition), the MBC used a two-tiered approach to ensure algorithm adherence. The two tiers included (1) the dissemination and implementation of decision support tools at the point of care and (2) a technology-based PFS.

Dissemination and implementation of decision support tools at the point of care. Once the STAR ${ }^{\star} \mathrm{D}$ protocol was agreed upon, the following specific steps were undertaken: (1) definition of critical points to guide pharmacotherapy decisions, (2) identification of brief clinical tools to monitor symptoms and side effects at each treatment visit, and (3) training of physicians and CRCs on the tools and critical decision points. These procedures aimed to maximize the internal validity of the study (ie to ensure that nonremission 
could be attributed to lack of efficacy and not to inadequate quality of care).

For each study medication, the decision support tools helped to increase the dosage to reach the maximally tolerated dose within the dose ranges mandated by $S T A R{ }^{*} D$, manage side effects adequately so that appropriate dosage increases were possible, and declare treatment failure if remission had not been reached after an adequate dose (maximally tolerated) and duration (up to 14 weeks). Treatment failure was determined by the 16-item Quick Inventory of Depressive Symptomatology-Clinician-rated (QIDS-C 16 ) (Rush et al, 2003b; Trivedi et al, 2004c).

The $S_{T A R}{ }^{\star D}$ clinical procedures manual (www.star-d. org) provided an overview of the MBC to optimize antidepressant treatment (Figure 1). It also specified the recommended dose increase based on the level of symptoms as determined by scores on the QIDS- $\mathrm{C}_{16}$ completed by the $\mathrm{CRC}$ at each treatment visit, and the exact target dosage(s) expected to be reached at specified times for each medication used in acute-phase treatment (Figure 2). Flexibility was built into the protocol regarding when to raise dosages or when to move to the next level or to followup. The manual required that participants be moved to the subsequent $\mathrm{STAR}^{\star} \mathrm{D}$ treatment level if they did not reach remission (QIDS- $\mathrm{C}_{16} \leqslant 5$ ) at the critical decision points of weeks 9 and 12 for each study medication, or were unable to tolerate side effects at any time point (with the proviso that side effects could be pharmacologically managed if appropriate).

Clinic visits occurred on weeks 0 (baseline), 2, 4, 6, 9, and 12. If the participant moved to the next level at any time during those visits, the schedule was started over with the last visit from the previous level counting as week 0 . The participant completed self-reports before being seen by the CRC. These included the self-report version of the QIDS $_{16}$ (the QIDS-SR ${ }_{16}$ ) and the following side-effect measures: the Frequency, Intensity, and Burden of Side Effects Rating (FIBSER) (Wisniewski et $a l, 2006)$ and Patient-Rated Inventory of Side Effects (Rush et al, 2004a). The CRC administered the QIDS- $\mathrm{C}_{16}$ and discussed any symptoms and side effects that the participant may have been experiencing. The CRC completed the appropriate sections of the case report form (CRF) using the information obtained from the participant. The CRFs were specifically constructed to obtain and record all necessary clinical information for easy decision-making. The CRFs were also designed to obtain pertinent clinical rationale when there were deviations from the algorithm (Figure 3 ).

The CRC ensured that each provider adhered to the treatment guidelines and took the appropriate steps at the critical decision points. Using the Clinical Procedures Manual guidelines for treatment, the CRC determined the appropriate treatment recommendation according to participant symptomatic response and tolerability to the

STAR $\star$ D Treatment
At-a-Glance

Figure I Overview of MBC for depression. 
CDP, Week 4

STAR $\star D$ Level 1

Symptom Improvement (SEs tolerable)

\begin{tabular}{|c|c|}
\hline QIDS-C $16 \geq 9$ & Increase dose to $40 \mathrm{mg} /$ day. \\
\hline QIDS-C ${ }_{16}=6-8$ & $\begin{array}{l}\text { Continue current dose, or } \\
\text { Increase dose to } 40 \mathrm{mg} / \text { day. }\end{array}$ \\
\hline QIDS-C ${ }_{16} \leq 5$ & Continue current dose. \\
\hline Improved, but SEs are intolerable & $\begin{array}{l}\text { Continue current dose and address SEs, or } \\
\text { Go to the next level. }\end{array}$ \\
\hline $\begin{array}{l}\text { Not improved and SEs are } \\
\text { intolerable }\end{array}$ & Go to the next level. \\
\hline Return to clinic: & Return in 2 weeks. \\
\hline $\begin{array}{l}\text { CDP, Week } 6 \\
\text { Symptom Improvement (SEs tolera }\end{array}$ & STAR $*$ D Level 1 \\
\hline QIDS- $\mathrm{C}_{16} \geq 9$ & Increase dose to $60 \mathrm{mg} /$ day. \\
\hline QIDS-C $16=6-8$ & $\begin{array}{l}\text { Increase dose to } 60 \mathrm{mg} / \text { day, or } \\
\text { Continue current dose. }\end{array}$ \\
\hline QIDS-C ${ }_{16} \leq 5$ & Continue current dose. \\
\hline Improved, but SEs are intolerable & $\begin{array}{l}\text { Continue current dose and address SEs, or } \\
\text { Decrease dose and continue for } 2 \text { additional weeks, or } \\
\text { Go to the next level. }\end{array}$ \\
\hline $\begin{array}{l}\text { Not improved and SEs are } \\
\text { intolerable }\end{array}$ & Go to the next level. \\
\hline Return to clinic: & Return in 3 weeks. \\
\hline
\end{tabular}

\begin{tabular}{|c|c|}
\hline CDP, Week 9 & STAR $*$ D Level 1 \\
\hline \multicolumn{2}{|c|}{ Symptom Improvement (SEs tolerable): } \\
\hline QIDS-C ${ }_{16} \geq 9$ & Go to the next level. \\
\hline QIDS-C $C_{16}=6-8$ & $\begin{array}{l}\text { Increase dose to } 60 \mathrm{mg} / \text { day, if not done previously, or } \\
\text { Go to next level. }\end{array}$ \\
\hline QIDS-C $16 \leq 5$ & Continue current dose. \\
\hline SEs are intolerable & Go to the next level. \\
\hline Return to clinic: & Return in 3 weeks. \\
\hline CDP, Week 12 & STAR $*$ D Level 1 \\
\hline \multicolumn{2}{|c|}{ Symptom Improvement (SEs tolerable): } \\
\hline QIDS-C ${ }_{16} \geq 9$ & Go to the next level. \\
\hline QIDS-C $\mathrm{C}_{16}=6-8$ & $\begin{array}{l}\text { Continue current dose and go to follow-up, or } \\
\text { Go to the next level. }\end{array}$ \\
\hline QIDS-C ${ }_{16} \leq 5$ & Continue current dose and go to follow-up. \\
\hline SEs are intolerable & Go to the next level. \\
\hline Return to clinic: & $\begin{array}{l}\text { If remitted, } \\
\text { - Return in } 3 \text { months or as needed. } \\
\text { If starting new level, } \\
\text { - Return in } 2 \text { weeks. }\end{array}$ \\
\hline
\end{tabular}

Figure 2 Critical decision points for level I treatment.

medication. Before each clinician visit, the CRC gave the CRF to the clinician and discussed the protocol-based treatment recommendations with the clinician. The clinician then met with the participant, made a final treatment determination, and completed the relevant sections of the CRF (including the Clinical Global Impression and noting the prescribed study medication).

Technology-based physician feedback system. The PFS was an online reporting system available through the $S T A R^{\star} D$ Web site that allowed clinicians easy access to view their level of adherence to the protocol. Physicians and the CRC would $\log$ on to a secure Web site with access to their own patients. The site included a prompting and feedback system that provided specific alerts when treatment deviated from the algorithm. This interactive system provided resources and guidelines to busy clinicians to help ensure a maximally tolerated dose and duration for each step in the algorithm and was available following the treatment visit.

The PFS was generated from the clinic visit documentation system, based on the compiled information from CRFs completed at each participant visit (Figure 3). It included depressive symptom severity (ie the scores on the QIDS- $\mathrm{C}_{16}$ and QIDS-SR 16 at the visit, and the QIDS- $\mathrm{C}_{16}$ score at baseline), dose of the study medication, and the FIBSER. These data elements were faxed to the STAR*D Data Center and were similar to the information used in a computerized 


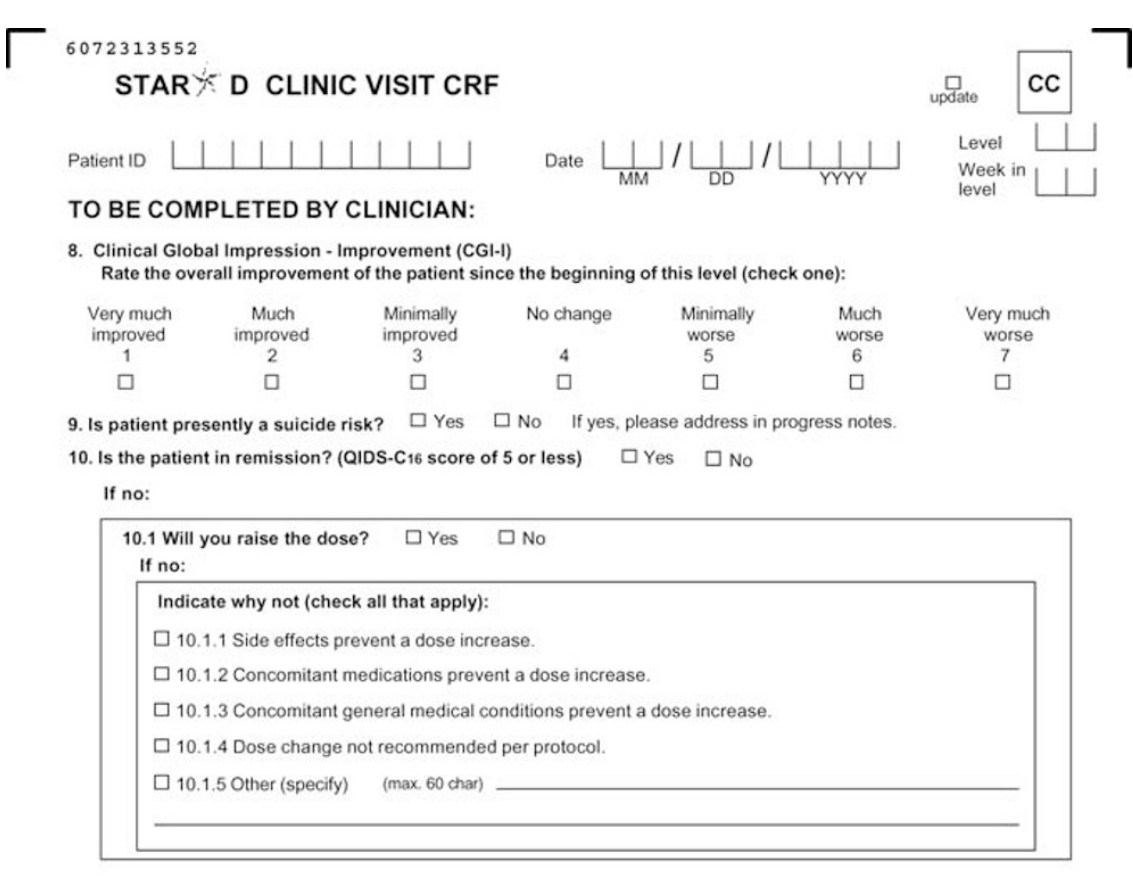

11. Have study medications or doses changed at this visit? This includes stop date(s) for discontinued meds.

$\square$ Yes (specify) $\square$ No

\begin{tabular}{|c|c|c|c|c|}
\hline CODE' & $\begin{array}{c}\text { MEDICATION NAME } \\
\text { (please print) }\end{array}$ & DAILY DOSE* & $m m \quad{ }_{d d}^{\text {START DATE }}{ }_{y y y y}$ & 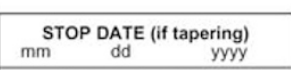 \\
\hline$\sqcup \sqcup \sqcup$ & & $\sqcup \sqcup \perp \perp . \sqcup \bigsqcup_{\mathrm{mg}}$ & ப」/し」し」」 & ப」/し」/し」」 \\
\hline$\sqcup \sqcup \sqcup$ & & $\sqcup \perp \perp \perp . \sqcup \mathrm{mg}$ & ப」/し」し」」」 & $\sqcup \sqcup / \sqcup \sqcup / \sqcup \sqcup \perp$ \\
\hline 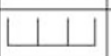 & & $\sqcup \perp \perp \perp . \sqcup \bigsqcup_{\mathrm{mg}}$ & 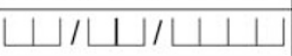 & 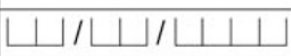 \\
\hline 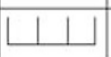 & & $\sqcup \perp\left|\downarrow . \sqcup \sqcup_{\mathrm{mg}}\right|$ & 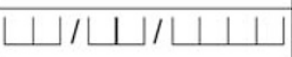 & ப」/し/し」」 \\
\hline$\sqcup \sqcup \sqcup$ & & $\sqcup \sqcup \perp \sqcup . \sqcup_{\mathrm{mg}}$ & 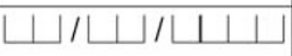 & ப」/し/し」」 \\
\hline$\sqcup \sqcup \sqcup$ & & $\sqcup \sqcup \sqcup \sqcup . \sqcup_{\mathrm{mg}} \mid$ & ப」/ப/し」ل」 & பل/し/し」」 \\
\hline$\cdot$ See & ation code list & Record Thyroid in mcg. & & \\
\hline
\end{tabular}

Figure 3 Case report form (includes dose escalation and side-effect monitoring).

decision system (Trivedi et al, 2004a). This information was entered into the database and reported on a participant-byparticipant and visit-by-visit basis on the web page of the clinical monitoring portion of the $\mathrm{STAR}^{\star} \mathrm{D}$ web site. An example of the alert system display for one subject is provided in Table 1. These reports were posted on the web within $48 \mathrm{~h}$ of the data having entered the database and were displayed by clinical site using confidential participant ID numbers. Physicians and CRCs were asked to access reports, which provided quick visual tables of the entire series of visits for each participant being treated by a specific physician. Based on these reports, physicians or CRCs could arrange for appropriate changes in dose for the participant, and a dose escalation could be accomplished without having to wait for a subsequent clinical visit. This system was designed to allow for corrective action at the treating clinician level, although it often required CRCs to provide reminders to treating physicians. The Data Center and the National Coordinating Center staff could also access these reports for the entire study population.
The online monitoring system included an easy-to-use online alert system. The alert system enhanced rapid feedback and allowed corrective action, where appropriate, without burdening the clinicians and CRCs. A special computer program was developed encompassing the following data: (1) current dose of the particular study medication, (2) how long the participant had been on the current dose, (3) total QIDS- $\mathrm{C}_{16}$ score at the time of the visit, (4) scores on the FIBSER, and most importantly (5) the recommendations from the critical decision table for the particular study medication at the specified treatment in the protocol. On the basis of this information, the Web-based report displayed flags highlighting violations of specific decision rules at each treatment visit. These violations included (1) continuing the same medication and not moving to the subsequent level when it was recommended, (2) dosing too high (indicating that the study medication was increased at a rate more rapid than recommended), (3) not addressing side effects, (4) dosing too low (ie an increased dose of medication was indicated), (5) moving to 
Table I Web-Based Reports for Sample Patient

\begin{tabular}{|c|c|c|c|c|c|c|c|c|}
\hline Patient ID & Level & Week & Date & Clinician & QIDS-C $_{16}$ & GRSEB & $\mathbf{R x}$ & Alert \\
\hline I234567ABC & । & 0 & $06 / 17 / 02$ & Smith & 19 & - & CIT 20 & $O K^{a}$ \\
\hline I234567ABC & I & 2 & $07 / 01 / 02$ & Smith & 18 & 4 & CIT 40 & Address SEs \\
\hline $1234567 A B C$ & I & 4 & $07 / 22 / 02$ & Smith & II & 2 & CIT 60 & Dose too high \\
\hline $1234567 A B C$ & । & 6 & 08/05/02 & Smith & 8 & 0 & CIT 60 & $O K^{\mathrm{a}}$ \\
\hline $1234567 A B C$ & I & 9 & $08 / 19 / 02$ & Smith & 12 & 0 & CIT 60 & Continued tx \\
\hline
\end{tabular}

Abbreviations: GRSEB, Global Rating of Side Effects Burden; QIDS-C 16, 16-item Quick inventory of Depressive Symptomatology; SEs, side effects; Tx, treatment.

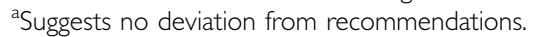

Table 2 Frequency of Web-Based Alerts for Level I

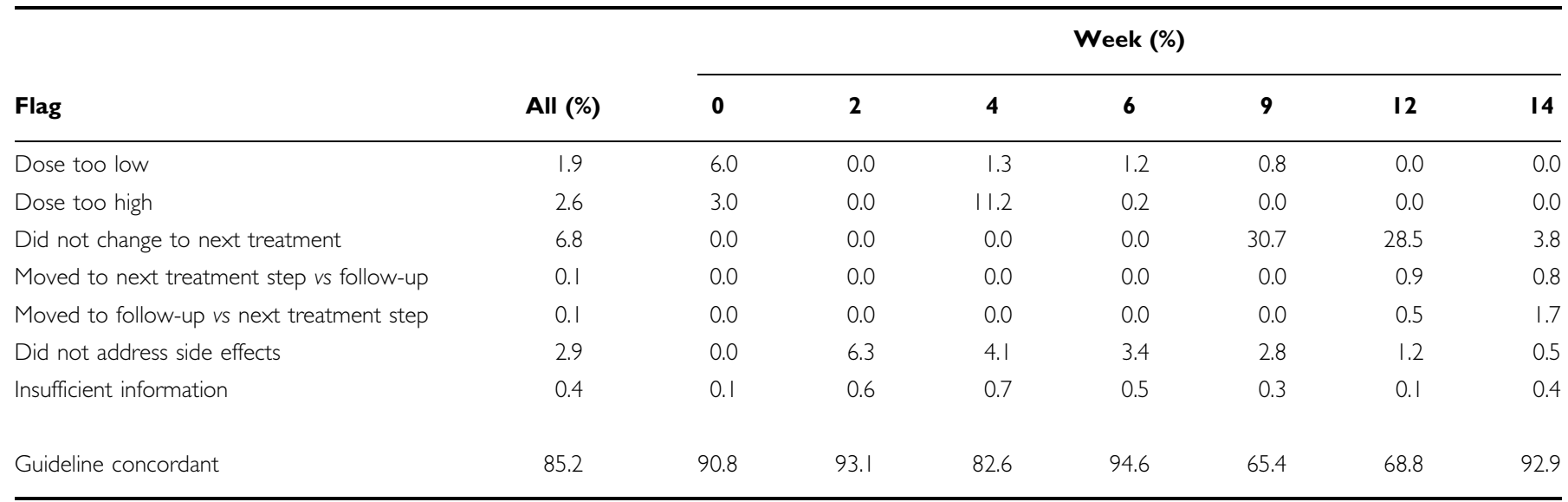

the next level (before the completion of an adequate trial), and (6) moving to long-term follow-up too soon. The flags represented cases in need of review, although often an appropriate clinical rationale for the decision made was available on that review. The reports were updated every Monday, Wednesday, and Friday.

The following specific rules were used to develop online alert flags:

Alert/Flag 1: Did not change treatment stage (continued treatment). A decision to move to the next treatment level or to long-term follow-up was recommended based on the protocol and clinical status, but the participant had been continued on the same treatment regimen. This flag suggested that the participant was receiving a treatment that had proven to be ineffective for a long enough duration, and a new treatment was indicated.

Alert/Flag 2: Dose too high. The dose of the antidepressant medication was increased at a faster rate than recommended or was dosed above the highest dose permitted by the protocol. This flag indicated that the current dose for the specified duration of antidepressant exposure was too high.

Alert/Flag 3: Side effects not addressed. The side-effect burden rating was significant and a medication adjustment or randomization to the next treatment level was indicated, but no changes had been made. The Global Rating of Side Effects Burden is a 0-6-point rating system. A score of 4, 5, or 6 was deemed to be significant and indicated the need to address the side effects either by adjusting the dose of the antidepressant medication, by treating the side effect, or by randomizing the participant to the next treatment level.

Alert/Flag 4: Dose too low. The dose of the antidepressant medication had been increased at a slower rate than recommended. This flag indicated that the participant was in danger of receiving suboptimal dosing for the medication in spite of the lack of significant side effects.

Alert/Flag 5: Moved to subsequent step even when not indicated. This flag indicated that the participant had been taken to the next level when the algorithm indicated the participant should have moved to long-term follow-up.

Alert/Flag 6: Moved to follow-up too soon. This flag indicated that the participant had been moved to long-term follow-up when the protocol recommended movement to the next treatment level.

This monitoring system also provided useful summaries of physician adherence during the study for use by the physicians and study leadership. Table 2 shows the distribution of the frequency of flags generated from the use of citalopram at level 1 . The system generated a surprisingly low nonadherence rate of $15 \%$, suggesting that $85 \%$ of visits were within the specific guideline/protocol requirements (4041 participants; mean: 3.48 visits). Many of the deviations had an acceptable rationale given the complex clinical characteristics of the participant population. For example, most flags involved extending the treatment trial at 9 and/or 12 weeks although the protocol 
suggested moving to the next level of treatment. These deviations, however, helped ensure that a fully adequate treatment trial was implemented, and by 14 weeks decisions were protocol concordant again.

Regular teleconference calls were also used to ensure a smoother transfer of information and to facilitate treatment adherence. These calls provided specific feedback relevant to the most common deviations from the recommended treatment procedures, as well as discussion among the study physicians regarding the most recent relevant literature.

\section{COMMENT}

The successful implementation of the protocol, and the initial findings from use of the MBC system with level 1 citalopram treatment in $\mathrm{STAR}^{\star} \mathrm{D}$ suggest that it is feasible to implement a flexible and yet rational system for successful $\mathrm{MBC}$ and to provide helpful feedback to physicians conducting antidepressant treatment. The system addressed all the medications in the algorithm and maximized the delivery of optimal antidepressant treatment in terms of dose, duration, and management of side effects. At the same time, it allowed the flexibility that is vital when treating complex, difficult-to-treat patients with medical and psychiatric comorbidities in busy primary and specialty care practices. The system, including the measures of symptom severity, side effects, and dosing recommendations, is easy to use and can be easily translated into clinical practice. At both levels 1 and 2 in STAR ${ }^{\star} \mathrm{D}$ (Rush et al, 2006; Trivedi et al, 2006a, b), the quality of care was enhanced by the system. Patients averaged approximately five visits over 10 weeks of treatment with vigorous antidepressant dosing that, on balance, met or exceeded moderate dosing levels (Fava, 2003), suggesting that an adequate treatment exposure was achieved with each of the medications in first two steps of the $\operatorname{STAR}^{\star} \mathrm{D}$ algorithm. Accordingly, wider use of the $\mathrm{MBC}$ approach in routine psychiatric and primary care is likely to enhance quality of care and patient outcomes for MDD.

The disparity between 'best practice' and actual practice in the treatment of depression has been well documented (Bauer, 2002; Crown et al, 2001; Schulberg et al, 1997). Ornstein et al (2000), in a study of 389 primary care physicians, also found that there is a wide variation in prescribing antidepressants in primary care. These problems tend to occur in both primary and psychiatric care settings. Studies indicate that the recovery rates of depressed patients treated in the primary care setting are lower than those anticipated from treatments consistent with the Agency for Healthcare Research and Quality (AHRQ) guidelines. Less than half the patients (30-40\%) receive treatments as recommended by the AHRQ guidelines. A study by Young et al (2001) found that only 19\% of patients with depression in primary care received appropriate treatment. Patients are often undertreated with respect to dose and duration of antidepressant medication; only two-fifths of the depressed patients treated in primary care reach the recommended therapeutic dosage, and only one-third continue to take the antidepressant medication past the first month. The follow-up and frequency of return visits are also inadequate, particularly during the critical initial stages of treatment. Schulberg et al (1999) found that patients were seen about 2-4 times within 8 weeks, which is about half of what was recommended during the initial critical 6-8-week period. The result is that optimal outcomes (full symptomatic remission and return of premorbid levels of functioning) are often not achieved.

The success of even the most well-developed protocols has been very limited in real-world practice (Cabana et al, 1999, 2002; Trivedi et al, 2002). Further, even effective protocols have not been sufficient to meet patient needs in routine clinical care owing to Axis I and III comorbidities. It is vital for clinicians to have a systematic prompt and feedback system to monitor the adequacy of treatment on an ongoing basis because feedback at the point of care is often necessary to maximize delivery of effective care. In $\mathrm{STAR}^{\star} \mathrm{D}$, this innovative $\mathrm{MBC}$ approach that included the use of CRFs, routine measurement of symptoms and side effects, and 'hands on' assistance from CRCs was used to enhance adherence to treatment recommendations at the point of care. An automated feedback system complemented the point of care system following the treatment visit.

This approach may help bridge the gap between controlled trials and real-world practice by providing a clear gain to both patient and physician. For physicians, the benefit is a flexible, nonintrusive support and monitoring system that takes minimal professional time and guides, but does not force, ongoing management of care based on clinically meaningful measures. By specifically reviewing depressive symptoms and side effects, $\mathrm{MBC}$ provides a context, already generally used for other chronic illnesses, for more comprehensively reviewing the patient's response to treatment - a key aid in the busy real-world management of depression in psychiatric and primary care settings. Also, the system increases the likelihood of adequate treatment trials maximizing the probability of the best outcomes; this approach produced mean antidepressant doses higher than most primary care and psychiatric physicians usually prescribe (Trivedi et al, 2006b). For patients, the approach enhances their understanding of the goals and objectives of treatment, and helps them monitor progress objectively and participate in an informed manner in decisions about their care.

If successful in future studies, this or other comparable measurement- and feedback-based systems can be incorporated in routine clinical care practice and provide a generalizable method of implementing evidence-based treatment algorithms. Many large clinical centers utilize computerized health information systems that enhance clinician decision-making. These include the University of Iowa (Flanagan et al, 1995), the University of Minnesota Hospital Clinic's Web Clinical Information System (Willard et al, 1995), Kaiser Permanente's San Diego Department of Preventive Medicine Interactive Health Appraisal System (Aydin et al, 1995), and Columbia-Presbyterian Medical Center's Medical Entities Dictionary (Cimino et al, 1995). Similarly, automated clinical care protocols have been used in England (Herbert et $a l, 1995)$ and The Netherlands (Vissers et al, 1996a, b). The clinical practice setting has yet to incorporate the computer as a tool for clinical decisionmaking (Flanagan et al, 1995; Ireland, 1996; Rotman et al, 1996). Problems remain in existing software that deter 
physician acceptance, including physician downtime for data entry (Hammer et al, 1995), nonstandardized codes for clinician and computer interfacing (Johnson, 1996), perceived problems of maintaining patient and provider confidentiality (Immonen, 1996), protection of records integrity from data hackers (Von Solms, 1996), lack of portability of computer modules (Werner et al, 1995), and poorly designed workstations (Dayhoff et al, 1994).

Some attempts have been made in various medical fields to implement algorithms via computer systems. Implementation, acceptance, and adherence are generally more successful in settings where computerized systems, such as computerized medical record systems or computerized physician orders, are already in place (Litzelman et al, 1993; Margolis et al, 1992; McDonald et al, 1984; Tierney et al, 1995). A major benefit of computerizing an algorithm is the increased interface between physician and algorithm. The prompting of the computer enables the physician to receive the information more effectively and respond accordingly. Owing to their complexity, most algorithms are improperly utilized, particularly in the absence of a systematic feedback loop. Computerizing an algorithm with feedback will help the physician to assimilate the information correctly and respond effectively. Subsequent analyses will address the impact of varying degrees of algorithm fidelity on patientlevel outcomes (Trivedi et al, 2000, 2002, 2004a).

In summary, the following areas of concern persist in the delivery of state-of-the-art pharmacotherapy for depression: adequate dose levels, monitoring of symptoms, side-effect burden and function, duration of treatment, follow-up care and frequency of return visits, and wide variation in care. Our MBC, which is automated and nonintrusive, is designed to address these concerns. It signals a paradigm shift to the use of research tools that maximize quality pharmacotherapy in routine clinical practice.

\section{ACKNOWLEDGEMENTS}

This project was funded with Federal funds from the National Institute of Mental Health, National Institutes of Health, under Contract N01MH90003 to UT Southwestern Medical Center at Dallas (PI: AJ Rush). Dr Trivedi was supported in part by the NIMH 5R01MH067692-2 (Treatment With Exercise Augmentation for Depression (TREAD)) and 5R01MH064062-2 (Computerized Decision Support System for Depression (IMPACTS)).

The content of this publication does not necessarily reflect the views or policies of the Department of Health and Human Services, nor does mention of trade names, commercial products, or organizations imply endorsement by the US Government. We thank the editorial support of Jon Kilner, MS, MA, and the secretarial support of Fast Word Information Processing Inc. (Dallas, Texas).

\section{THE STAR ${ }^{\star D}$ STUDY INVESTIGATORS}

National Coordinating Center: AJ Rush, MD; MH Trivedi, MD; D Warden, PhD, MBA; MM Biggs, PhD; K ShoresWilson, PhD; D Stegman, RN; M Kashner, PhD, JD.

Data Coordinating Center: S Wisniewski, PhD; GK Balasubramani, PhD; J Luther, H Eng, BA.
$S T A{ }^{\star} D$ Regional Centers: Alabama: University of Alabama, Birmingham, AL and Tuscaloosa Veterans Affairs Medical Center, Tuscaloosa, AL (L Davis, MD; K Rice, MSW; A Berry, RN; P Johnson, MSN; S Ambrose, RN; M Jewell, MA; B Thomas, MSW; E Waldrop, RNC; T Allen, BA; E St John, BA; R Williams, PharmD).

California: University of California, Los Angeles, CA (A Leuchter, MD; I Lesser, MD; I Cook, MD; M Epstein, RN, NP; S Rosenberg, MN, RN, CNS; S Zeim, BS; L Sulkowski, BS; J Iribarren, PsyD; R Armstrong, BS; A Rosales, MA; M Abrams, BSN).

California: University of California, San Diego, CA (S Zisook, MD; K Harless, BA; C Gonzalez, MD; M Smith, RN; C Lawrence, MFS; J Palica, MD; M Rohrs, BA; M CapousDesyllas, MSW; K Ganadjian, MD).

Illinois: Northwestern University Medical School, Chicago, IL (W McKinney, MD; W Gilmer, MD; C Kelley, RNC; C Cooler, RN; A Bauer, MA; J Fleck, MN, RN, CNP; C Endick, MSW).

Kansas: Psychiatric Research Institute, University of Kansas, Wichita, KS (S Preskorn, MD; D Hilger, LPN; A Klick-Davis, BSN; R Lusk, BSN; J Elmore, BSN; D Soetaert, BA).

Massachusetts: Massachusetts General Hospital, Boston, MA (J Alpert, MD; M Fava, MD; A Nierenberg, MD; A Farabaugh, $\mathrm{PhD} ; \mathrm{T}$ Petersen, $\mathrm{PhD} ; \mathrm{W}$ Merens, MA; $\mathrm{P}$ Cassano, MD; N Craven, MD; H Yang, MD; M Candrian, MS; R Fraguas, MD, $\mathrm{PhD}$ ).

Michigan: University of Michigan, Ann Arbor, MI (E Young, MD; S Marcus, MD; J Greden, MD; H Briggs, MSW; K Bullard, MSW, MPH; A Kennedy, BS; A Benway, BS; E Rickard, BA).

New York: New York State Psychiatric Institute and Columbia College of Physicians and Surgeons, New York, NY (FM Quitkin, MD; P McGrath, MD; JW Stewart, MD; H Sackeim, PhD; K Tate-Brown, MS, NPP, CCRC; S Rees, RN, MSN, CS; C Smith, MS, CS, NPP; A Couraud, BSN, RN; J Lavelle, MS; K Broderick, BSW).

North Carolina: University of North Carolina, Chapel Hill, NC (R Golden, MD; Bradley Gaynes, MD; J DeVeaugh-Geiss, MA, LPA; A Ford, MA; S Barnett, MEd; B Pearson, PLCSW).

Oklahoma: Laureate Healthcare System, Tulsa, OK (J Mitchell, MD; W Yates, MD; J Kuehnert, RN, CCRC; L Jernigan, RN; B Williams, MS, RN; J Hilton, RN).

Pennsylvania: University of Pittsburgh Medical Center, Pittsburgh, PA (M Thase, MD; RH Howland, MD; E Friedman, MD; J Callan, RN, MSN; S Berman, MEd; L Shutt, RN, MSN; C Spotts, MEd).

Tennessee: Vanderbilt University Medical Center, Nashville, TN (S Hollon, PhD; R Shelton, MD; M Lovett, MEd; T Crutcher, RN; T Patton, MA; J Hart, BS; RM Harris-Turner, RN, MSN; D Lilly, MSSW; B Sirles, MPH; S Hicks, RN, MSN; N Harris, High School; S Addington, MA).

Texas: The University of Texas Southwestern Medical Center, Dallas, TX (M Husain, MD; M Downing, MD; D Stegman, RNC; E Shellhorn, BA; B O’Neal, RN, BSN; D Turner, RN; L MacLeod, RN, CCRC; M Henson, RN, BSN; T Hawley, RN, BSN, CCRC; S Gardner, MS).

Virginia: Virginia Commonwealth University, Richmond, VA (S Kornstein, MD; R Schneider, MD; S Belyea PhD; B Perry, MS; K Schmitt, BA; T Goff, LCSW, CCRC; K Lamoree, MA; M Britton, RN, BSN; C Glassman, BS). 


\section{REFERENCES}

American Psychiatric Association (2000). Diagnostic and Statistical Manual of Mental Disorders, 4th edn, Text Revision. American Psychiatric Press: Washington, DC.

Aydin CE, Rosen PN, Jewell SM, Felitti VJ (1995). Computers in the examining room: the patient's perspective. Proc Annu Symp Comput Appl Med Care 824-828.

Bauer MS (2002). A review of quantitative studies of adherence to mental health clinical practice guidelines. Harv Rev Psychiatry 10: $138-153$.

Biggs MM, Shores-Wilson K, Rush AJ, Carmody TJ, Trivedi MH, Crismon ML et al (2000). A comparison of alternative assessments of depressive symptom severity: a pilot study. Psychiatry Res 96: 269-279.

Cabana MD, Rand CS, Powe NR, Wu AW, Wilson MH, Abboud PA et al (1999). Why don't physicians follow clinical practice guidelines? A framework for improvement. JAMA 282: $1458-1465$

Cabana MD, Rushton JL, Rush AJ (2002). Implementing practice guidelines for depression: applying a new framework to an old problem. Gen Hosp Psychiatry 24: 35-42.

Cimino JJ, Johnson SB, Hripcsak G, Hill CL, Clayton PD (1995). Managing vocabulary for a centralized clinical system. Medinfo 8(Part 1): 117-120.

Crown WH, Treglia M, Meneades L, White A (2001). Long-term costs of treatment for depression: impact of drug selection and guideline adherence. Value Health 4: 295-307.

Davis LL, Rush AJ, Wisniewski SR, Rice K, Cassano P, Jewell ME et al (2005). Substance use disorder comorbidity in major depressive disorder: an exploratory analysis of the Sequenced Treatment Alternatives to Relieve Depression cohort. Compr Psychiatry 46: 81-89.

Dayhoff R, Kirin G, Pollock S, Miller C, Todd S (1994). Medical data capture and display: the importance of clinicians' workstation design. Proc Annu Symp Comput Appl Med Care 541-545.

Fava M (2003). Diagnosis and definition of treatment-resistant depression. Biol Psychiatry 53: 649-659.

Fava M, Rush AJ, Trivedi MH, Nierenberg AA, Thase ME, Sackeim HA et al (2003). Background and rationale for the sequenced treatment alternatives to relieve depression $\left(\mathrm{STAR}^{\star} \mathrm{D}\right)$ study. Psychiatr Clin North Am 26: 457-494, x.

Flanagan JR, Olson JD, Wagner JR, Nuehring NE, Krumbholz SD (1995). Cost-effective health information systems: user-driven internal development at the University of Iowa. Proc Annu Symp Comput Appl Med Care 703-707.

Ford DE (2000). Managing patients with depression: is primary care up to the challenge? J Gen Intern Med 15: 344-345.

Hamilton M (1960). A rating scale for depression. J Neurol Neurosurg Psychiatry 23: 56-62.

Hamilton M (1967). Development of a rating scale for primary depressive illness. Br J Soc Clin Psychol 6: 278-296.

Hammer JS, Strain JJ, Friedberg A, Fulop G (1995). Operationalizing a bedside pen entry notebook clinical database system in consultation-liaison psychiatry. Gen Hosp Psychiatry 17: $165-172$.

Herbert SI, Gordon CJ, Jackson-Smale A, Salis JL (1995). Protocols for clinical care. Comput Methods Programs Biomed 48: 21-26.

Immonen S (1996). Developments in health care, the increasing role of information technology: security issues. Int J Biomed Comput 43: 9-15.

Ireland RS (1996). Initial experience of the ORATEL computerised information tools in general practice. Br Dent J 181: 289-295.

Johnson SB (1996). Generic data modeling for clinical repositories. J Am Med Inform Assoc 3: 328-339.

Katon W, Von Korff M, Lin E, Walker E, Simon GE, Bush T et al (1995). Collaborative management to achieve treatment guidelines. Impact on depression in primary care. JAMA 273: 1026-1031.

Katon W, Von Korff M, Lin E, Simon G, Walker E, Unutzer J et al (1999). Stepped collaborative care for primary care patients with persistent symptoms of depression: a randomized trial. Arch Gen Psychiatry 56: 1109-1115.

Katon WJ, Von Korff M, Lin EH, Simon G, Ludman E, Russo J et al (2004). The pathways study: a randomized trial of collaborative care in patients with diabetes and depression. Arch Gen Psychiatry 61: 1042-1049.

Kessler RC, Berglund P, Demler O, Jin R, Koretz D, Merikangas KR et al (2003). The epidemiology of major depressive disorder: results from the National Comorbidity Survey Replication (NCS-R). JAMA 289: 3095-3105.

Kobak KA, Greist JH, Jefferson JW, Katzelnick DJ (1996). Computer-administered clinical rating scales: a review. Psychopharmacology 127: 291-301.

Kobak KA, Greist JH, Jefferson JW, Mundt JC, Katzelnick DJ (1999). Computerized assessment of depression and anxiety over the telephone using interactive voice response. $M D$ Comput 16: 63-68.

Lin EHB, Von Korff M, Katon W, Bush T, Simon GE, Walker E et al (1995). The role of the primary care physician in patients' adherence to antidepressant therapy. Med Care 33: 67-74.

Litzelman DK, Dittus RS, Miller ME, Tierney WM (1993). Requiring physicians to respond to computerized reminders improves their compliance with preventive care protocols. J Gen Intern Med 8: 311-317.

Margolis CZ, Warshawsky SS, Goldman L, Dagan O, Wirtschafter D, Pliskin JS (1992). Computerized algorithms and pediatricians' management of common problems in a community clinic. Acad Med 67: 282-284.

McDonald CJ, Hui SL, Smith DM, Tierney WM, Cohen SJ, Weinberger $M$ et al (1984). Reminders to physicians from an introspective computer medical record. A two-year randomized trial. Ann Intern Med 100: 130-138.

Mundt JC (1997). Interactive voice response systems in clinical research and treatment. Psychiatr Serv 48: 611-612.

Ornstein S, Stuart G, Jenkins R (2000). Depression diagnoses and antidepressant use in primary care practices: a study from the Practice Partner Research Network (PPRNet). J Fam Pract 49: 68-72.

Rotman BL, Sullivan AN, McDonald TW, Brown BW, DeSmedt P, Goodnature D et al (1996). A randomized controlled trial of a computer-based physician workstation in an outpatient setting: implementation barriers to outcome evaluation. J Am Med Inform Assoc 3: 340-348.

Rush AJ (1999). Linking efficacy and effectiveness research in the evaluation of psychotherapies. In: Miller NE, Magruder KM (eds). Cost Effectiveness of Psychotherapy. A Guide for Practitioners, Researchers and Policymakers. Oxford University Press: New York, NY. pp 26-32.

Rush AJ, Crismon ML, Kashner TM, Toprac MG, Carmody TJ, Trivedi MH et al (2003a). Texas medication algorithm project, phase 3 (TMAP-3): rationale and study design. J Clin Psychiatry 64: 357-369.

Rush AJ, Fava M, Wisniewski SR, Lavori PW, Trivedi MH, Sackeim HA et al (2004a). Sequenced treatment alternatives to relieve depression $\left(\mathrm{STAR}^{\star} \mathrm{D}\right)$ : rationale and design. Control Clin Trials 25: 119-142.

Rush AJ, Koran LM, Keller MB, Markowitz JC, Harrison WM, Miceli RJ et al (1998). The treatment of chronic depression, part 1: study design and rationale for evaluating the comparative efficacy of sertraline and imipramine as acute, crossover, continuation, and maintenance phase therapies. J Clin Psychiatry 59: $589-597$.

Rush AJ, Trivedi M, Carmody TJ, Biggs MM, Shores-Wilson K, Ibrahim $\mathrm{H}$ et al (2004b). One-year clinical outcomes of 
depressed public sector outpatients: a benchmark for subsequent studies. Biol Psychiatry 56: 46-53.

Rush AJ, Trivedi MH, Ibrahim HM, Carmody TJ, Arnow B, Klein $\mathrm{DN}$ et al (2003b). The 16-item quick inventory of depressive symptomatology (QIDS), clinician rating (QIDS-C), and selfreport (QIDS-SR): a psychometric evaluation in patients with chronic major depression. Biol Psychiatry 54: 573-583; Erratum, p 585.

Rush AJ, Trivedi MH, Wisniewski SR, Stewart JW, Nierenberg AA, Thase ME et al (2006). Bupropion-SR, sertraline, or venlafaxine-XR after failure of SSRIs for depression. $N$ Engl J Med 354: 1231-1242.

Rush AJ, Zimmerman M, Wisniewski SR, Fava M, Hollon SD, Warden D et al (2005). Comorbid psychiatric disorders in depressed outpatients: demographic and clinical features. J Affect Disord 87: 43-55.

Schulberg HC, Block MR, Madonia MJ, Scott CP, Lave JR, Rodriguez E et al (1997). The 'usual care' of major depression in primary care practice. Arch Fam Med 6: 334-339.

Schulberg HC, Katon WJ, Simon GE, Rush AJ (1999). Best clinical practice: guidelines for managing major depression in primary medical care. J Clin Psychiatry 60(Suppl 7): 19-26.

Simon GE, VonKorff M, Wagner EH, Barlow W (1993). Patterns of antidepressant use in community practice. Gen Hosp Psychiatry 15: $399-408$

Tierney WM, Overhage JM, Takesue BY, Harris LE, Murray MD, Vargo DL et al (1995). Computerizing guidelines to improve care and patient outcomes: the example of heart failure. J Am Med Inform Assoc 2: 316-322.

Trivedi MH, Baker SM (2001). Clinical significance of monitoring early symptom change to predict outcome. J Clin Psychiatry 62(Suppl 4): 27-33.

Trivedi MH, Fava M, Wisniewski SR, Thase ME, Quitkin F, Warden D et al (2006a). Medication augmentation after the failure of SSRIs for depression. N Engl J Med 354: 1243-1252.

Trivedi MH, Kern JK, Baker SM, Altshuler KZ (2000). Computerizing medication algorithms and decision support systems for major psychiatric disorders. J Psychiatr Pract 6: 237-246.

Trivedi MH, Kern JK, Grannemann BD, Altshuler KZ, Sunderajan $\mathrm{P}$ (2004a). A computerized clinical decision support system as a means of implementing depression guidelines. Psychiatr Serv 55: 879-885.

Trivedi MH, Kern JK, Marcee A, Grannemann B, Kleiber B, Bettinger $\mathrm{T}$ et al (2002). Development and implementation of computerized clinical guidelines: barriers and solutions. Methods Inf Med 41: 435-442.

Trivedi MH, Kleiber BA (2001). Using treatment algorithms for the effective management of treatment-resistant depression. J Clin Psychiatry 62: 25-29.
Trivedi MH, Rush AJ, Crismon ML, Kashner TM, Toprac MG, Carmody TJ et al (2004b). Clinical results for patients with major depressive disorder in the Texas Medication Algorithm Project. Arch Gen Psychiatry 61: 669-680.

Trivedi MH, Rush AJ, Ibrahim HM, Carmody TJ, Biggs MM, Suppes $\mathrm{T}$ et al (2004c). The inventory of depressive symptomatology, clinician rating (IDS-C) and Self-Report (IDS-SR), and the quick inventory of depressive symptomatology, clinician rating (QIDS-C) and self-report (QIDS-SR) in public sector patients with mood disorders: a psychometric evaluation. Psychol Med 34: 73-82.

Trivedi MH, Rush AJ, Wisniewski SR, Nierenberg AA, Warden D, Ritz L et al (2006b). Evaluation of outcomes with citalopram for depression using measurement-based care in $S_{T A R}{ }^{*} \mathrm{D}$ : implications for clinical practice. Am J Psychiatry 163: 28-40.

Unützer J, Katon W, Callahan CM, Williams Jr JW, Hunkeler E, Harpole L et al (2002). Collaborative care management of latelife depression in the primary care setting: a randomized controlled trial. JAMA 288: 2836-2845.

Vissers MC, Biert J, van der Linden CJ, Hasman A (1996a). Effects of a supportive protocol processing system (ProtoVIEW) on clinical behaviour of residents in the accident and emergency department. Comput Methods Programs Biomed 49: 177-184.

Vissers MC, Hasman A, van der Linden CJ (1996b). Impact of a protocol processing system (ProtoVIEW) on clinical behaviour of residents and treatment. Int J Biomed Comput 42: 143-150.

Von Solms B (1996). Managing secure computer systems and networks. Int J Biomed Comput 43: 47-52.

Werner R, Haux R, Leiner F, Winter A (1995). An integrated approach for mobile information processing in hospitals. Medinfo 8(Part 1): 395-399.

Willard KE, Hallgren JH, Sielaff B, Connelly DP (1995). The deployment of a World Wide Web (W3) based medical information system. Proc Annu Symp Comput Appl Med Care 771-775.

Wisniewski SR, Rush AJ, Balasubramani GK, Trivedi $\mathrm{MH}$, Nierenberg AA, for the STAR ${ }^{\star} D$ Investigators (2006). Self-rated global measure of the frequency, intensity, and burden of side effects. J Psychiatr Pract 12: 71-79.

Yates WR, Mitchell J, Rush AJ, Trivedi MH, Wisniewski SR, Warden D et al (2004). Clinical features of depressed outpatients with and without co-occurring general medical conditions in STAR ${ }^{*}$ D. Gen Hosp Psychiatry 26: 421-429.

Young AS, Klap R, Sherbourne CD, Wells KB (2001). The quality of care for depressive and anxiety disorders in the United States. Arch Gen Psychiatry 58: 55-61. 